\title{
Research Square \\ Gravitational cells. The formula of the gravitational constant. Calculation of the value of the gravitational constant in the region of a black hole.
}

Andrey Chernov ( $\sim$ and8591@gmail.com )

none Company/Institution

Article

Keywords: Black hole, gravitational cells, gravitational field, elementary charge, Schwarzschild radius formula, gravitational constant formula, gravitational constant, gravitational cell mass

Posted Date: June 17th, 2021

DOI: https://doi.org/10.21203/rs.3.rs-622706/v1

License: (c) (1) This work is licensed under a Creative Commons Attribution 4.0 International License.

Read Full License 


\section{Gravitational cells as a source of the gravitational field.}

The formula of the gravitational constant. Calculation of the value of the gravitational constant in the region of a black

hole.

\section{Author Andrey Chernov}

Address: Andrey Chernov, 16 Polenova str, apt.7, Irkutsk, 664007, Russia. cell phone: +7 9645407 298. E mail: and8591@gmail.com

\section{Content}

1. Abstract -2 pg.

2. Introduction $-3 \mathrm{pg}$.

3. Methods $-3-10 \mathrm{pg}$.

4. Results and discussion $-10-12$ pg.

5. Conclusions $-12-13 \mathrm{pg}$.

6. Declarations $-14 \mathrm{pg}$. 


\section{Abstract.}

In this study, a new concept is introduced into physics - gravitational cells. These cells are densely compressed elementary particles: a proton and an electron. The body of a black hole consists of a huge number of such cells.

On this theoretical basis, using the Schwarzschild radius formula and the adapted Coulomb formula, a formula for the gravitational constant was obtained and its value in the gravitational field of black holes was calculated, $\boldsymbol{G}_{\mathbf{0}}=\mathbf{6}, \mathbf{7 9 2 7}$. $\mathbf{1 0}^{-\mathbf{1 1}}$. Also, scientific substantiation of the value of the usual gravitational constant $\boldsymbol{G}$ was obtained.

In this study, a new physical constant was determined - the mass of the gravitational cell of a black hole $\boldsymbol{m}_{\mathbf{0}}=\mathbf{1 , 5 1 1 5 9 3} \cdot \mathbf{1 0}^{-27} \mathbf{k g}$.

Based on the results of the study, conclusions were drawn regarding the gravitational mass of the proton and the electron.

Keywords. Black hole, gravitational cells, gravitational field, elementary charge, Schwarzschild radius formula, gravitational constant formula, gravitational constant, gravitational cell mass. 


\section{Introduction.}

In the history of physics, there have been numerous attempts to obtain physically and mathematically substantiated formulas for calculating the gravitational constant. These proposed formulas for calculating the gravitational constant are quite complex (the degree of numbers in these formulas can reach 17), and also have many additional components. As a rule, in these formulas there is no elementary charge (except for the formula of Arthur Lunn). But at the same time in absolutely all formulas there is mass in the form of an electron, a nucleon, etc. But the mass (bodies and particles) from the relativistic point of view is a relative value, and therefore the authors of these formulas had to introduce artificial components into their formulas in order to obtain the final result $\boldsymbol{G}=\mathbf{6 , 6 7 4 3} \cdot \mathbf{1 0}^{\mathbf{- 1 1}}$.

This is the main significant point that distinguishes the formula obtained by the author for the gravitational constant in the region of the black hole $\boldsymbol{G}_{\mathbf{0}}=\sqrt{\mathbf{2}} \boldsymbol{q}_{\mathbf{0}} \boldsymbol{c}$, where there is no mass at all. On the basis of this formula, the value of the gravitational constant in the region of the black hole was calculated $\boldsymbol{G}_{\mathbf{0}}=\mathbf{6}, \mathbf{7 9 2 7}$.

$10^{-11}$. And only after that the value of the usual gravitational constant $G=$ $6,6743 \cdot 10^{-11}$ was scientifically substantiated and determined.

\section{Methods.}

The research should start with the Coulomb formula. Let's write the Coulomb formula for the case of interaction of two opposite elementary charges: 


$$
\boldsymbol{f}=\boldsymbol{k} \cdot \frac{q_{0} \cdot q_{0}}{r^{2}} \quad \text { or } \quad \boldsymbol{f}=\boldsymbol{k} \cdot \frac{q_{0}{ }^{2}}{r^{2}}
$$

Where $\boldsymbol{f}$ is the force of attraction of two elementary charges.

where $\boldsymbol{q}_{\mathbf{0}}$ is an elementary charge, $1,60217733 \cdot 10^{-19} \mathrm{C}$.

$\boldsymbol{K}$ is the coefficient of proportionality, $K=\frac{\mathbf{1}}{\mathbf{4 \pi \varepsilon _ { 0 } \varepsilon}}$. Where $\frac{\mathbf{1}}{\mathbf{4 \pi \varepsilon _ { 0 }}}$ is a constant equal to $\mathbf{9}$

$\cdot \mathbf{1 0}^{9} \frac{\mathrm{kg} \mathrm{m}}{\mathrm{C}^{2} \mathrm{~s}^{2}}$, and $\boldsymbol{\varepsilon}$ is the relative dielectric constant of the medium.

$\boldsymbol{r}$ is the distance between charges, $\mathrm{m}$.

The value of the proportionality coefficient $\boldsymbol{K}$ depends on the medium.

Maximum value $\boldsymbol{K}=\mathbf{9} \cdot \mathbf{1 0}^{9} \frac{\mathrm{kg} \mathrm{m}}{\mathrm{C}^{2} \mathrm{~s}^{2}}$ in vacuum (where $\boldsymbol{\varepsilon}=1$ ), for alcohol $\boldsymbol{K}=2,6$.

$10^{8}$ (where $\boldsymbol{\varepsilon}=35$ ), for water $\boldsymbol{K}=1,1 \cdot 10^{8}$ (where $\boldsymbol{\varepsilon}=80$ ), for copolymers $\boldsymbol{K} \geq 10^{4}$

(where $\boldsymbol{\varepsilon} \leq 105$ ), etc. Theoretically, $\boldsymbol{K}$ can approach its minimum value equal to $\mathbf{1}$.

But $\mathrm{k}<1$ cannot be in any medium, because then the fractionality of elementary

charges is allowed. Practical confirmation of this lies in the fact that science does not

know substances where $\boldsymbol{\varepsilon}>\boldsymbol{9} \cdot \mathbf{1 0}^{\mathbf{9}}$. The maximum value of $\boldsymbol{\varepsilon}$ is recorded for metal nanoisland structures, $10^{7}-10^{8}$, where $\boldsymbol{K} \geq 90$.

Now let's move on to the gravitational interaction of such physical objects as black holes. (The result obtained here will then make it possible to pass to the gravitational interaction of ordinary bodies).

So, we have two black holes of mass $\boldsymbol{M}$ and $\boldsymbol{M}_{\mathbf{1}}$, located at a distance $\mathrm{r}$ from each other. The body of a black hole consists of many superdense cells with mass $\boldsymbol{m}_{\mathbf{0}}$ and charge $\boldsymbol{q}_{\boldsymbol{g}}$, numerically equal to the value of two elementary charges $\boldsymbol{q}_{\mathbf{0}}$, 
that is, $\boldsymbol{q}_{\boldsymbol{g}}=\mathbf{2}_{\boldsymbol{q}_{\mathbf{0}}}$. Such a cell (let's call it a gravitational cell), in a black hole, is a tightly compressed pair of elementary particles: an electron and a proton.

In this case, the mass of the cell will be less than the total mass of a free proton and a free electron by the value $\Delta \boldsymbol{m}$ due to the release of energy, where $\Delta \boldsymbol{m}=$ $\boldsymbol{E} / \mathrm{c}^{2}$. The charges of the gravitational cells of a black hole create a common gravitational field $\boldsymbol{E}$ in space, through which they interact with the charges of the gravitational cells of another black hole. The charges of the gravitational cells of a black hole create a common gravitational field $\boldsymbol{E}$ in space, through which they interact with the charges of the gravitational cells of another black hole. The field strength of one gravitational cell $\boldsymbol{g}_{\mathbf{0}}$ of a black hole is determined by the following formula:

$$
g_{0}=\frac{k_{0} q_{g 1} \cdot k_{0} q_{g 2}}{r^{2}} \quad \text { or } \quad g_{0}=k_{0}^{2} \frac{4 q_{0}^{2}}{r^{2}}
$$

where $\boldsymbol{g}_{\mathbf{0}}$ is the value of the gravitational field of one gravitational cell of a black hole, $\mathrm{m} / \mathrm{s}^{2}$.

$\boldsymbol{k}_{\mathbf{0}}$ - proportionality coefficient, $\boldsymbol{k}_{\mathbf{0}}=\mathbf{1} \frac{\mathrm{kg}^{1 / 2} \mathrm{~m}^{3 / 2}}{\mathrm{C} \mathrm{s}}$,

$\boldsymbol{q}_{\mathbf{0}}$ - elementary charge $1,60217733 \cdot 10^{-19} \mathrm{C}$.

$\boldsymbol{q}_{\boldsymbol{g} \mathbf{1}}$ and $\boldsymbol{q}_{\boldsymbol{g} \mathbf{2}}$ are the charges of the gravitational cells of black holes, $\boldsymbol{q}_{\boldsymbol{g}}=\mathbf{2} \boldsymbol{q}_{\mathbf{0}}=$ $3,20435466 \cdot 10^{-19} \mathrm{C}$.

$\boldsymbol{r}$ - distance between cells, $\mathrm{m}$.

Thus, the gravitational field of one gravitational cell at a distance of $\boldsymbol{r}=\mathbf{1} \mathrm{m}$ will be $\boldsymbol{g}_{\mathbf{0}}=\mathbf{1 , 0 2 6 7 8 9} \cdot \mathbf{1 0}^{-37} \mathrm{~m} / \mathrm{s}^{2}$. 
Formula (1-2) is similar to Formula (1-1). But if in the Coulomb formula the coefficient of proportionality in vacuum is $\boldsymbol{k}=\mathbf{9} \cdot \mathbf{1 0}^{\mathbf{9}}$, then in the formula (1-2) $\boldsymbol{k}_{\mathbf{0}}=\mathbf{1}$. The reason for such a large discrepancy in the coefficients is that the substance of the black hole is so strongly compressed that almost all lines of force of elementary charges are closed inside gravitational cells. And only an extremely small part of the lines of force go out from the cell, creating a gravitational field in the outer space. As a result of this circumstance, the coefficient of proportionality of elementary charges outside the gravitational cell decreases to its minimum threshold, that is, exactly to 1. At the same time, the main electric field, with the coefficient of proportionality $\boldsymbol{k}=\mathbf{9} \cdot \mathbf{1 0}^{\mathbf{9}}$, remains closed between elementary charges inside the gravitational cells and therefore does not manifest itself in any way.

Now let's consider the interaction of two black holes $\boldsymbol{M}$ and $\boldsymbol{M}_{\mathbf{1}}$. The body of a black hole with mass $\boldsymbol{M}$ consists of a huge number of gravitational cells with mass $\boldsymbol{m}_{\mathbf{0}}$. Therefore, the total number of such cells will be: $\boldsymbol{n}=\frac{\boldsymbol{M}}{\boldsymbol{m}_{\mathbf{0}}}$. These gravitational cells form a common gravitational field in space, equal to $\boldsymbol{E}=\boldsymbol{g}_{\mathbf{0}} \cdot \boldsymbol{n}$. Thus, the gravitational field $\boldsymbol{E}$ of a black hole of mass $\boldsymbol{M}$ when interacting with another black hole $\boldsymbol{M}_{\mathbf{1}}$ located at a distance $\boldsymbol{r}$ will be:

$$
E=\frac{k_{0}^{2} 4 q_{0}^{2}}{r^{2}} \frac{M}{m_{0}} \quad \text { or } \quad E=\frac{k_{0}^{2} 4 q_{0}^{2}}{m_{0}} \frac{M}{r^{2}}
$$

The expression $\frac{\boldsymbol{k}_{\mathbf{0}}^{2} \boldsymbol{4 q}_{\mathbf{0}}{ }^{2}}{\boldsymbol{m}_{\mathbf{0}}}$ is the value of the gravitational constant $\boldsymbol{G}_{\mathbf{0}}$ for the case of interaction of two black holes. As a result, formula (1-3) will take the following form: 


$$
E=G_{0} \frac{M}{r^{2}}, \quad \text { где } \quad G_{0}=\frac{k_{0}^{2} 4 q_{0}^{2}}{m_{0}}
$$

It follows that $\boldsymbol{m}_{0}=\frac{\boldsymbol{k}_{0}^{2} \mathbf{4 q _ { 0 }}}{G_{0}}$. (Further, for a better perception of information, the coefficient $\boldsymbol{k}_{\mathbf{0}}$ will not be displayed in the formulas, due to the fact that $\boldsymbol{k}_{\mathbf{0}}=\mathbf{1}$ ). It is possible to determine the value $\boldsymbol{m}_{\mathbf{0}}$ if we admit the possibility of equality $\boldsymbol{G}_{\mathbf{0}}=$ $G=6,6743 \cdot 10^{-11}$. In this case, we get $m_{0}=\frac{4 q_{0}^{2}}{6,6743 \cdot 10^{-11}}=1,53842$. $\mathbf{1 0}^{-\mathbf{2 7}} \mathrm{kg}$. But such a calculation of the value $\boldsymbol{m}_{\mathbf{0}}$ cannot be considered correct, because the value of the gravitational constant $\boldsymbol{G}_{\mathbf{0}}$ in the region of the black hole may have a different value, that is, $\boldsymbol{G}_{\mathbf{0}} \neq \boldsymbol{G}$. Therefore, in order to correctly determine the value of $\boldsymbol{m}_{\mathbf{0}}$ and also the value of $\boldsymbol{G}_{\mathbf{0}}$, it is necessary to turn to the formula for the Schwarzschild gravitational radius:

$$
R=\frac{2 G_{0}}{c^{2}} \cdot M \quad(1-4)
$$

where $\boldsymbol{R}$ is the gravitational radius of a black hole, $\boldsymbol{G}_{\mathbf{0}}$ is the gravitational constant in the field of a black hole, $\boldsymbol{M}$ is the mass of a black hole, and $\boldsymbol{c}$ is the speed of light.

In this formula, of particular interest is the expression $\frac{2 G_{0}}{c^{2}}$, which is measured in "m / kg", and denotes the specific length of the gravitational radius of a black hole. (In what follows, we denote $\frac{2 G_{0}}{c^{2}}$ as $\boldsymbol{\gamma}$ ). And now a very important point. In space compressed to a point around a black hole, such a category as space does not exist. Therefore, in the Schwarzschild radius, all physical quantities merge into one quantity - mass, where the initial dimensional unit is the mass of the gravitational cell $\boldsymbol{m}_{\mathbf{0}}$. As a result of this circumstance, in the gravitational radius of the black hole, the 
numerical equality must be observed: $\boldsymbol{\gamma}=\boldsymbol{m}_{\mathbf{0}}$. Taking into account that $\boldsymbol{\gamma}=\frac{2 \boldsymbol{G}_{\mathbf{0}}}{\boldsymbol{c}^{2}}$, and $m_{0}=\frac{4 q_{0}^{2}}{G_{0}}$, we get the following equation: $\frac{2 G_{0}}{c^{2}}=\frac{4 q_{0}^{2}}{G_{0}}$. Let's solve it and as a result we get:

$$
\begin{aligned}
& G_{0}=\sqrt{2} q_{0} c=6,7927 \cdot 10^{-11} \frac{\mathrm{m}^{3}}{\mathrm{~s}^{2} \mathrm{~kg}} \\
& m_{0}=\frac{\sqrt{8} q_{0}}{c}=1,511593 \cdot 10^{-27} \mathrm{~kg}
\end{aligned}
$$

As you can see, $\boldsymbol{G}_{\mathbf{0}}$ differs from $\boldsymbol{G}=\mathbf{6 , 6 7 4 3} \cdot \mathbf{1 0}^{-\mathbf{1 1}}$ by $1,7 \%$. In this case, the value of $\boldsymbol{G}_{\mathbf{0}}$ is related to the mass of the gravitational cell $\boldsymbol{m}_{\mathbf{0}}$ by the following beautiful formula: $G_{0}=\frac{m_{0} c^{2}}{2}(\mathbf{1 - 5})$.

Now let's consider the gravitational interaction of an ordinary (that is, not superdense) body of mass $\boldsymbol{M}$ with another ordinary body of mass $\boldsymbol{M}_{\mathbf{1}}$. The formula for the gravitational field of the body $\boldsymbol{M}$ in this case will look like this:

$$
E=\frac{k^{2} 4 q_{0}^{2}}{r^{2}} \frac{M}{m}=\frac{k^{2} 4 q_{0}^{2}}{m} \frac{M}{r^{2}} \quad \text { or } \quad E=G \frac{M}{r^{2}}
$$

Where $\boldsymbol{k}$ is the proportionality coefficient of the charge of the gravitational cell of an ordinary body, where $\boldsymbol{k}=\mathbf{1}, \mathbf{0 3 8 9 3 1} \frac{\mathrm{kg}^{1 / 2} \mathrm{~m}^{3 / 2}}{\mathrm{C} \mathrm{s}}, \boldsymbol{k}^{2}=\mathbf{1 , 0 7 9 3 7 8} \frac{\mathrm{kg} \mathrm{m}^{3}}{\mathrm{C}^{2} \mathrm{~s}^{2}}$ $\boldsymbol{m}$ is the mass of the gravitational cell, where $\boldsymbol{m}=\mathbf{1 , 6 6 0 5 3 9} \cdot \mathbf{1 0}^{-\mathbf{2 7}} \mathrm{kg}$ $\boldsymbol{G}$ - gravitational constant, where $\boldsymbol{G}=\mathbf{6 , 6 7 4 3} \cdot \mathbf{1 0}^{-\mathbf{1 1}} \frac{\mathrm{m}^{3}}{\mathrm{~s}^{2} \mathrm{~kg}}$

Now let's explain the magnitude of $\boldsymbol{k}$ and $\boldsymbol{m}$. Let's start with $\boldsymbol{m}=$ $\mathbf{1 , 6 6 0 5 3 9} \cdot \mathbf{1 0}^{-\mathbf{2 7}} \mathrm{kg}$. To do this, imagine that two bodies of mass $\boldsymbol{M}$ and $\boldsymbol{M}_{\mathbf{1}}$ were formed from two black holes $\boldsymbol{M}^{\prime}$ and $\boldsymbol{M}^{\prime}{ }_{\mathbf{1}}$. In this case, each gravitational cell of the 
black hole, due to the arrival of energy $\boldsymbol{E}$, increases its mass $\boldsymbol{m}_{\mathbf{0}}$ to mass $\boldsymbol{m}$ by the amount $\Delta \boldsymbol{m}$ (where $\Delta \boldsymbol{m}=\boldsymbol{E} / \mathrm{c}^{2}$ ). As a result, a plasma is formed from the superdense matter of a black hole, from which gaseous, liquid and solid substances can be formed. All four states of matter are neutral, that is, they have a total electric charge equal to zero. As a result of this circumstance, any substance can be represented as a huge set of gravitational cells. These cells consist of a proton and an electron with a total charge of $\mathbf{2} \boldsymbol{q}_{\mathbf{0}}$, as well as of neutrons, which are also a pair of a proton and an electron with a total charge of $\mathbf{2} \boldsymbol{q}_{\mathbf{0}}$. Thus, the mass of the gravitational cell $\boldsymbol{m}$ of any non-superdense substance (plasma, gas, liquid and solid) with high accuracy will be equal to $\mathbf{1} \mathbf{D a}$ (this is $1 / 12$ of the mass of an atom of the isotope of carbon-12) or $\mathbf{1 , 6 6 0 5 3 9} \cdot \mathbf{1 0}^{\mathbf{- 2 7}} \mathrm{kg}$. This implies that:

$$
k=\sqrt{\frac{G m}{4 q_{0}^{2}}}=\sqrt{\frac{6,6743 \cdot 10^{-11} m}{4 q_{0}^{2}}}=1,038931
$$

Thus, $\boldsymbol{k}>\boldsymbol{k}_{\mathbf{0}}$. From a physical point of view, this can be explained by the fact that, in contrast to the gravitational cell of a black hole, in an ordinary cell there is some very small distance between elementary charges. As a result of this circumstance, more lines of force come out of an ordinary cell and go out, creating a $7,9 \%$ stronger gravitational field in outer space. But due to the fact that the mass of an ordinary gravitational cell $\boldsymbol{m}$ is more than the mass of the gravitational cell of a black hole $\boldsymbol{m}_{\mathbf{0}}$ by $9.9 \%$, then $1 \mathrm{~kg}$ of ordinary matter has a smaller number of gravitational cells than $1 \mathrm{~kg}$ of black hole matter. As a result of this circumstance, it turns out that $\boldsymbol{G}<\boldsymbol{G}_{\mathbf{0}}$, even though $\boldsymbol{k}>\boldsymbol{k}_{\mathbf{0}}$. For clarity, we will show this in a mathematical form: 


$$
G=\frac{k^{2} 4 q_{0}^{2}}{m}=\frac{1,079378 \cdot 4 q_{0}^{2}}{1,660539 \cdot 10^{-27}}<G_{0}=\frac{k_{0}^{2} 4 q_{0}^{2}}{m_{0}}=\frac{4 q_{0}^{2}}{1,511593 \cdot 10^{-27}}
$$

Next, consider the gravitational interaction of a black hole with mass $\boldsymbol{M}_{\mathbf{0}}$ and an ordinary body with mass $\boldsymbol{M}$ at a distance $\boldsymbol{r}$ between them.

1. The gravitational field $\boldsymbol{E}$ of a black hole with mass $\boldsymbol{M}_{\mathbf{0}}$ will be:

$$
E=\frac{k_{0} k 4 q_{0}^{2}}{r^{2}} \frac{M_{0}}{m_{0}}=\frac{k_{0} k 4 q_{0}^{2}}{m_{0}} \frac{M_{0}}{r^{2}} \text { (1-7) or } E=G_{1} \frac{M_{0}}{r^{2}}
$$

where $\boldsymbol{k}_{\mathbf{0}}=\mathbf{1}, \boldsymbol{k}=\mathbf{1}, \mathbf{0 3 8 9 3 1}, \boldsymbol{m}_{\mathbf{0}}=\mathbf{1}, \mathbf{5 1 1 5 9 3} \cdot \mathbf{1 0}^{-27} \mathrm{~kg}$. Hence the gravitational constant is equal to:

$$
G_{1}=\frac{k_{0} k 4 q_{0}^{2}}{m_{0}}=\frac{1,038931 \cdot 4 q_{0}^{2}}{1,511593 \cdot 10^{-27}}=7,0572 \cdot 10^{-11} \frac{\mathrm{m}^{3}}{\mathrm{~s}^{2} \mathrm{~kg}}
$$

2. The gravitational field $\boldsymbol{E}$ of an ordinary body of mass $\boldsymbol{M}$ will be:

$$
E=\frac{k_{0} k 4 q_{0}^{2}}{r^{2}} \frac{M}{m}=\frac{k_{0} k 4 q_{0}^{2}}{m} \frac{M}{r^{2}}(1-8) \text { or } E=G_{2} \frac{M}{r^{2}}
$$

where $\boldsymbol{k}_{\mathbf{0}}=\mathbf{1}, \boldsymbol{k}=\mathbf{1}, \mathbf{0 3 8 9 3 1}, \boldsymbol{m}_{\mathbf{0}}=\mathbf{1}, \mathbf{5 1 1 5 9 3} \cdot \mathbf{1 0}^{-27} \mathrm{~kg}$. Hence the gravitational constant is equal to:

$$
G_{2}=\frac{k_{0} k 4 q_{0}^{2}}{m}=\frac{1,038931 \cdot 4 q_{0}^{2}}{1,660539 \cdot 10^{-27}}=6,4242 \cdot 10^{-11} \frac{\mathrm{m}^{3}}{\mathrm{~s}^{2} \mathrm{~kg}}
$$

\section{Results and discussion.}

As a result of the study, gravitational constants were determined for different cases of gravitational interaction.

1. $\boldsymbol{G}_{\mathbf{0}}=\mathbf{6}, \mathbf{7 9 2 7} \cdot \mathbf{1 0}^{-\mathbf{1 1}}$ (for the case of interaction of two black holes).

2. $\boldsymbol{G}=\mathbf{6}, \mathbf{6 7 4 3} \cdot \mathbf{1 0}^{-\mathbf{1 1}}$ (for the case of interaction of two ordinary bodies). 
3. $\boldsymbol{G}_{\mathbf{1}}=\mathbf{7}, \mathbf{0 5 7 2} \cdot \mathbf{1 0}^{-11}$ and $\boldsymbol{G}_{\mathbf{2}}=\mathbf{6}, \mathbf{4 2 4 2} \cdot \mathbf{1 0}^{-\mathbf{1 1}}$ (for the case of interaction between a black hole and an ordinary body).

Another significant scientific result of this study is the determination of the mass of the gravitational cell of a black hole $\boldsymbol{m}_{\mathbf{0}}=\mathbf{1}, \mathbf{5 1 1 5 9 3} \cdot \mathbf{1 0}^{-\mathbf{2 7}} \mathrm{kg}$.

Before proceeding to the discussion of the results, it is necessary to explain the third case of gravitational interaction, where there are two values of the gravitational constants $\boldsymbol{G}_{\mathbf{1}}$ and $\boldsymbol{G}_{\mathbf{2}}$. The presence of two gravitational constants may raise the question: "Isn't there a violation of Newton's third law?" There is no violation here, because throughout the study, the mass of bodies was only a measure of the amount of matter, and nowhere did it act as a gravitational or inertial mass. This is clearly seen from formulas (1-7) and (1-8), in which the masses $\boldsymbol{M}_{\mathbf{0}}$ and $\boldsymbol{M}$ show only the number $\mathrm{n}$ of gravitational cells in the body $\boldsymbol{n}=\frac{\boldsymbol{M}_{\mathbf{0}}}{\boldsymbol{m}_{\mathbf{0}}}$ and $\boldsymbol{n}=\frac{\boldsymbol{M}}{\boldsymbol{m}}$ and nothing more. Therefore, Newton's third law $\left(\boldsymbol{F}_{\mathbf{1}}=-\boldsymbol{F}_{\mathbf{2}}\right)$, where there is inertial mass $\left(\boldsymbol{m}_{\mathbf{1}} \boldsymbol{a}_{\mathbf{1}}=\right.$ $-\boldsymbol{m}_{\mathbf{2}} \boldsymbol{a}_{\mathbf{2}}$ ), cannot be applied here. Therefore, there is no contradiction in the fact that in one gravitational interaction there are two gravitational constants.

When discussing the results of the study, it is necessary to pay attention to the following important point. The force of interaction between two elementary charges $\boldsymbol{q}_{\mathbf{0}}$ inside the gravitational cell is relatively large and is determined by the formula (11), where the proportionality coefficient is $\boldsymbol{k}=\mathbf{9} \cdot \mathbf{1 0}^{\mathbf{9}}$. But outside the gravitational cell, the proportionality coefficient $\boldsymbol{k}$ decreases sharply and for superdense gravitational cells it becomes equal to $\mathbf{1}$, for ordinary gravitational cells $\mathbf{- 1 , 0 3 8 9 3 1}$. 
This circumstance $\mathbf{9} \cdot \mathbf{1 0}^{9}$ times weakens the electric field outside the gravitational cell, turning it into a gravitational field.

\section{Conclusions.}

An important result of this research is the derivation of the formula for the gravitational constant and the determination of its value for different variants of the gravitational interaction (see the section "Results").

It should be specially noted here that the gravitational constant for the interaction of two black holes was obtained by the formula $\boldsymbol{G}_{\mathbf{0}}=\sqrt{\mathbf{2}} \boldsymbol{q}_{\mathbf{0}} \boldsymbol{c}$. Thus, when calculating the value $G_{0}=6,7927 \cdot 10^{-11}$, only two independent fundamental constants were used: the elementary charge $q_{0}$ and the speed of light $\boldsymbol{c}$ ). Thus, when calculating the value $\boldsymbol{G}_{\mathbf{0}}=\mathbf{6 , 7 9 2 7} \cdot \mathbf{1 0}^{-\mathbf{1 1}}$, only two independent fundamental constants were used: the elementary charge $\boldsymbol{q}_{\mathbf{0}}$ and the speed of light $\boldsymbol{c}$, in which the order of the numbers differs by $\mathbf{1 0}^{\mathbf{2 7}}$ times. Therefore, from the standpoint of probability theory, the $\mathbf{1 , 7 \%}$ difference between $\boldsymbol{G}_{\mathbf{0}}$ and $\boldsymbol{G}$ confirms the correctness of the result obtained.

In this study, the mass of the gravitational cell of a black hole was determined, where $\boldsymbol{m}_{\mathbf{0}}=\mathbf{1}, \mathbf{5 1 1 5 9 3} \cdot \mathbf{1 0}^{-\mathbf{2 7}} \mathrm{kg}$. The value of the gravitational constant in the region of interaction of black holes depends on the value $\boldsymbol{m}_{\mathbf{0}}$, which can be determined by the following formula: $\boldsymbol{G}_{\mathbf{0}}=\frac{\boldsymbol{m}_{0} \boldsymbol{c}^{2}}{2}$. At the same time, there are no gravitational cells where the mass is less. Thus, based on the above, $\boldsymbol{m}_{\mathbf{0}}$ can be 
classified as a new physical constants. At the same time, the gravitational constant has ceased to be an independent physical constant, because theoretically it can have several values.

Based on the results obtained in this study, it can be concluded that the source of the gravitational field is the gravitational cells, which consist of two elementary particles (proton and electron). Separately, a proton and an electron do not have gravitational mass and therefore cannot create a gravitational field. It is impossible to prove the opposite experimentally. Because to measure on a torsion-balance, you have to combine at least 1 kilogram of protons or electrons together. And this is completely impossible due to the repulsion of the same charges. And this is completely impossible due to the repulsion of identical charges. In contrast, in this study, based on the hypothesis of gravitational cells, the value of the gravitational constant in the gravitational field of black holes was accurately calculated. At the same time, the calculations were based on the formula $\boldsymbol{G}_{\mathbf{0}}=\mathbf{6}, \mathbf{7 9 2 7} \cdot \mathbf{1 0}^{-\mathbf{1 1}}$, where such a concept as gravitational mass is completely absent.

A more detailed interpretation of the results obtained in this study will be given later. Now at this stage, it is important to emphasize that these results cannot be accidental and to continue further research. 


\section{Declarations}

1. Availability of data and materials.

All data obtained and analyzed in the course of this study is included in this article.

2. Competing interests. Not applicable (there are no competing interests).

3. Funding. Not applicable.

4. Authors' contributions. Not applicable.

5. Acknowledgements. Not applicable. 